\title{
Post-perihelion observations of comet 1P/Halley
}

\author{
$\mathrm{V}: r_{\mathrm{h}}=28.1 \mathrm{AU}{ }^{\star}$ \\ O. R. Hainaut ${ }^{1}$, A. Delsanti ${ }^{2}$, K. J. Meech ${ }^{3}$, and R. M. West ${ }^{4}$ \\ 1 European Southern Observatory, Casilla 19001, Santiago 19, Chile \\ 2 Observatoire de Paris-Meudon - LESIA, 5 Place Jules Janssen, 92195 Meudon Cedex, France \\ e-mail: Audrey.Delsanti@obspm.fr \\ 3 Institute for Astronomy, University of Hawai' $i, 2680$ Woodlawn Drive, Honolulu, HI 96822, USA \\ e-mail: meech@ifa.hawaii.edu \\ ${ }^{4}$ European Southern Observatory, Karl-Schwarzschild-Straße 2, 85748 Garching-bei-München, Germany \\ e-mail: rwest@eso.org
}

Received 11 November 2003 / Accepted 18 December 2003

\begin{abstract}
Deep CCD observations have been performed simultaneously with the three $8.2 \mathrm{~m}$ Very Large Telescopes at Paranal, in the direction of comet 1P/Halley, on March 6-8, 2003. The comet, at heliocentric distance $r=28.1 \mathrm{AU}$, was convincingly detected (at $S / N=8$ ) on a composite of 32284 s exposure, as a point-source located $1.4^{\prime \prime}$ from the expected position. The object is also visible (at the $S / N=3-5$ level) on independent per-night or per-instrument composites, confirming the reality of the object, and the match of the observed motion over 3 consecutive nights with the predicted motion of 1P/Halley guarantees that this cannot be another Solar System object. The magnitude of the object, $R=28.22 \pm 0.13$, is compatible with the average cross-section of the bare nucleus.
\end{abstract}

Key words. comets: individual: 1P/Halley

\section{Introduction}

This is the fifth paper in the series describing the monitoring of comet $1 \mathrm{P} /$ Halley after perihelion, carried out at the European Southern Observatory since 1988. The earlier papers concern observations at heliocentric distance $r=8.5 \mathrm{AU}$ (West \& Jørgensen 1989, Paper I), $r=10.1$ (West 1990, Paper II), at $r=12.5 \mathrm{AU}$ and during the major outburst at $r=14.3 \mathrm{AU}$ (West et al. 1991, Paper III), at $r=16.6$ and 18.8 AU (Hainaut et al. 1995, Paper IV).

These observations of 1P/Halley were obtained in our long term program aimed at (i) characterizing the cometary activity at large heliocentric distances (e.g. Meech 1991, 1999; Meech et al. 2000; Hainaut et al. 2000; Meech \& Hainaut 2001) and (ii) determining the comet nucleus surface properties and size distribution (e.g. Hainaut et al. 1998; Meech 2003; Hainaut $\&$ Delsanti 2002; Meech et al. 2003). The direct goal of these observations was to perform a last check on the comet's status and activity, and to obtain a last set of astrometric measurements in order to secure the orbit for a future early pre-perihelion recovery.

\footnotetext{
Send offprint requests to: O. Hainaut, e-mail: ohainaut@eso.org

* Based on observations collected at the European Southern Observatory, Paranal, Chile, program 70.C-0198.
}

In Sect. 2, we describe the observations and data reduction, and in Sect. 3, we discuss the comet 1P/Halley results.

\section{Observations and data processing}

This monitoring was started on the $1.54 \mathrm{~m}$ Danish telescope on La Silla (out to $r=14.3 \mathrm{AU}$ ), continued on the $3.58 \mathrm{~m}$ New Technology Telescope, down to magnitude $V=26.5$ at $r=18.8 \mathrm{AU}$. For these observations, we used three of the four $8.2 \mathrm{~m}$ Unit Telescopes (UT 1, 3 and 4) of the ESO VLT on Paranal. UT 1 and 4, with FORS-1 and -2 respectively, were allocated simultaneously on 3 consecutive nights for another of our programs aimed at performing a very deep, ecliptic pencil-beam survey for Trans-neptunian Objects. The field of $1 \mathrm{P} /$ Halley was selected as a comparison field at higher ecliptic latitude, to be observed at the beginning of night while the main field was too low. The results of this TNO survey will be presented in another paper (Hainaut et al., in preparation). Table 1 lists the instruments, their parameters and filters used. VIMOS, on UT 3, was not yet released to the community; nevertheless, the observatory director allocated us some time on this instrument to test its capabilities for very deep imaging. FORS- 1 and -2 and VIMOS are described at http://www. eso.org/paranal/sciops. 
The filter (see Table 1) was selected in order to maximize the contrast between a comet nucleus with a typical color of $V-R=0.44$ (Hainaut \& Delsanti 2002) and the sky, and taking into account the response curves of the instruments. The FORS1 V is a standard Bessel filter. The "R-SPECIAL" filter on FORS2 is a Bessel filter whose passband has been slightly shortened on the red end in order to avoid sky emission lines. VIMOS' filter "vm-R-1.5" is essentially a Mould $R$ filter.

The total exposure time was obtained by accumulating individual exposures of 360 or $600 \mathrm{~s}$ (cf. Table 1). The telescopes were using the non-sidereal tracking rates of the comet (resulting in an elongation of the stars by $0.5-0.8^{\prime \prime}$ per frame). Between the exposures, the telescopes were offset by random shifts, pre-computed so that the comet always fell within $40^{\prime \prime}$ from the center (in order to avoid any systematic effect from the CCDs), and that the position of the background objects on the chips were always $>3^{\prime \prime}$ from their positions on the other exposures. The $\log$ of the observations is given in Table A.1 (including Moon and seeing) and summarized in Table 2. The conditions were photometric during the observations, with moderate $(3-6 \mathrm{~m} / \mathrm{s})$ North wind. The seeing was in the $0.6-0.9^{\prime \prime}$ range; on VIMOS, the automatic focussing of the instrument was not yet perfectly calibrated, resulting in degraded image quality $\left(0.9-1.6^{\prime \prime}\right)$.

The ephemerides of the comet were generated using orbital elements provided by B. G. Marsden for the epoch of the observations. Table 3 summarizes the geometric circumstances of the observations.

The frames have at first been processed in a standard way, using MIDAS (Banse et al. 1988; ESO 1999). A template bias (obtained by averaging many 0 -s exposures with a median rejection) has been subtracted from the raw data. The resulting frames have been divided by a flatfield obtained by averaging (with median rejection) several normalized twilight frames. We found that this procedure was not satisfactory for the low spatial frequencies. A second step of flatfielding was applied: each processed frame was normalized to an average sky level of 1 over a region clean of bad columns. The objects $2 \sigma$ above the sky noise were marked, and new "night sky flatfields" were obtained by averaging (with rejection of the marked area) these normalized frames. The images have been re-flatfielded using these new flats, resulting in a very uniform sky. The statistics of the sky area indicates that optimal flatfield was reached for all spatial scales.

The flatfielded frames were then processed with SExtractor (Bertin \& Arnout 1996), using the sky subtraction option, resulting in frames with sky at 0 . The catalogues of objects were searched for stars (using the magnitude, the stellarity index and the FWHM), and the seeing of each frame is obtained by averaging the FWHM of these stars (listed in Table A.1).

As the 3 instruments have different pixel scales (cf. Table 1) and slightly different orientations, we re-binned the FORS2 and VIMOS images to match the geometry of FORS1. This was done by building a template of the FORS1 data (by shift-and-add of the frames recentering on the background objects). On this template, 10 stars were identified surrounding the region where the comet is expected. The same stars were measured on each frame of FORS2 and VIMOS, and the
Table 1. Instrument characteristics Notes: 1- FORS2: only the "Master" chip was used for this program. A secondary chip is also available in the mosaic, constituting a total field of $6.6 \times 6.6^{\prime}$ with a narrow gap between them. 2- VIMOS: only the B1 chip was used for this program. In total, VIMOS has 4 similar cameras, imaging four fields with a gap of $2^{\prime}$ between them. The central wavelengths and bandwith [nm] of the filters are listed below their ESO designation. Exp. time refers to the individual exposure times.

\begin{tabular}{lccc}
\hline \hline Instrument & FORS-1 & FORS-2 & VIMOS \\
\hline Telescope & UT1 & UT4 & UT3 \\
Detector & TK2048EB & MIT/LL CCID & EEV CCD \\
& $4-1160$ & $20-14-5-3^{1}$ & $44-59 \mathrm{~B}^{2}$ \\
Pixel scale & $0.20^{\prime \prime}$ & $0.25^{\prime \prime}$ & $0.205^{\prime \prime}$ \\
Field & $6.8^{\prime}$ & $7^{\prime} \times 4^{1}$ & $6.7^{\prime} \times 7.2^{2}$ \\
Filter & V-BESS & R-SPECIAL & vm-R-1.5 \\
& $554 / 111.5$ & $655 / 165.0$ & $655 / 140$ \\
Exp. time & $600 \mathrm{~s}$ & $360 \mathrm{~s}$ & $360 \mathrm{~s}$ \\
\hline
\end{tabular}

rotation, translation and scaling parameters were computed using MIDAS' ALIGN/IMAGE command. We verified that this procedure produced a very good match of the transformed images with the template: the distortion is less than 0.1 pix $\left(0.02^{\prime \prime}\right)$ over the region crossed by the comet, and reaches a 0.3 pix inaccuracy in the outer regions of the CCD (this is caused by non-linear geometric distortions in the instruments). The FORS2 and VIMOS frames were also multiplied by a constant to match their Analog-to-Digital unit conversion factor to that of FORS1. At this stage, all the frames have virtually completely similar characteristics. When combining FORS-1 data (obtained in $V$ ) with those of the other instruments (through filter $R$ ), the typical $V-R=0.44$ color was used for the nucleus in order to correct the fluxes.

The field was calibrated astrometrically with POS1 (Walter \& West 1986) in its MIDAS implementation, using 45-69 stars from the USNO-A catalogue. In order to account for the second order distortion of the frames, each instrument was calibrated independently, using a $2 \times 5$ term transformation of coordinates (i.e. $x, y, x^{2}, y^{2}$ and $x y$, for both RA and Dec). The position of the comet in each frame was computed using the mid-exposure epoch, Marsden's ephemerides and the astrometric transformation.

In a first step, composite images were obtained by coadding the processed frames re-centered on the expected position for the comet. However, because of the very deep limiting magnitudes of the frames, many faint objects trail over the comet, making it hard to see. So, background templates were generated (one per instrument) by co-adding all the frames recentered on the stars. These templates were then subtracted from the individual frames. While the correction is not perfect because of seeing mis-match (we don't have enough frames to compute different templates per seeing bin), all the faint and fuzzy background objects (i.e. the large majority) completely disappear, as do the wings of brighter objects. Only the core 
Table 2. Summary of the observations and photometric measurements. For each combination of date(s) and telescope(s), the table lists: total exposure times [s]/number of frames and measured magnitude, per telescope and per night. In some cases, the object is not visible in the corresponding composite, or is visible, but too faint to be measured (less than $2 \sigma$ detection). The rejected frames (see text) are not included in these totals.

\begin{tabular}{lllll}
\hline \hline UT & FORS1 & FORS2 & VIMOS & All \\
Day & UT1 & UT4 & UT3 & \\
\hline 06 & $1200 \mathrm{~s} / 2$ & - & $3960 \mathrm{~s} / 11$ & $5160 \mathrm{~s} / 13$ \\
& not vis. & & $R=27.97 \pm 0.25$ & Visible \\
\hline 07 & $4800 \mathrm{~s} / 8$ & $3600 \mathrm{~s} / 10$ & $6124 \mathrm{~s} / 17$ & $14524 \mathrm{~s} / 35$ \\
& $V=28.19 \pm .56$ & $R=28.33 \pm 0.34$ & $R=28.28 \pm 0.20$ & $R=28.07 \pm 0.16$ \\
\hline 08 & $1800 \mathrm{~s} / 3$ & $3600 \mathrm{~s} / 10$ & $7200 \mathrm{~s} / 20$ & $12600 \mathrm{~s} / 33$ \\
& Visible & $R=27.83 \pm 0.17$ & $R=28.04 \pm 0.23$ & $R=28.05 \pm 0.16$ \\
\hline \multirow{2}{*}{ All } & $7800 \mathrm{~s} / 13$ & $7200 \mathrm{~s} / 20$ & $17284 \mathrm{~s} / 48$ & $32284 \mathrm{~s} / 81$ \\
& Visible & $R=27.92 \pm 0.15$ & $R=28.228 \pm 0.16$ & $R=28.22 \pm 0.13$ \\
\hline
\end{tabular}

Table 3. Geometric circumstances of the observations (left) and astrometric measurements performed on the nightly composite of all 3 telescopes (right). Epoch is the mid-exposure UT; $\alpha^{\prime}$ and $\delta^{\prime}$ are the apparent motions [arcsec/h] in RA and Dec; $r$ and $\Delta$ are the helio- and geocentric distances [AU]; $\beta$ is the solar phase angle [deg]. $\alpha$ and $\delta$ are the measured J2000. coordinates. The (O-C) are given in arcsec with respect to the ephemerides (B. Marsden, priv. comm.)

\begin{tabular}{lllllllllll}
\hline \hline Epoch & $\alpha^{\prime}$ & $\delta^{\prime}$ & $r$ & $\Delta$ & $\beta$ & $\alpha$ & $\delta$ & $(\mathrm{O}-\mathrm{C})_{\alpha}$ & $(\mathrm{O}-\mathrm{C})_{\delta}$ \\
\hline 2003-03-06.06487 & -4.51 & 2.17 & 28.07 & 27.26 & 1.20 & 084114.56 & -000323.6 & 1.2 & 1.2 \\
$2003-03-07.09644$ & -4.45 & 2.18 & 28.07 & 27.27 & 1.22 & 084107.09 & -000228.3 & 1.0 & 1.6 \\
$2003-03-08.08795$ & -4.39 & 2.19 & 28.07 & 27.28 & 1.25 & 084100.22 & -000136.7 & 1.2 & 1.2 \\
\hline
\end{tabular}

of brighter stars show up in the resulting frames. A second set of composite images were then obtained by co-adding these background subtracted frames, re-centered on the position of the comet.

\section{Results}

In Fig. 1, we display a 32284 s composite of all the frames, recentered on the predicted position of the comet. A point-like object is clearly visible close to the center (with $S / N=8$ ). Each individual frames was inspected, and those with a background object (verified on a star-centered composite) passing near or on that position were rejected, and new composites generated, with a similar result.

In order to verify the reality of the candidate, composites were also generated using various independent subsets of the data. The object is detected at the same position in many of these composites (cf. Table 2), confirming that (i) the object is real and (ii) its motion matches perfectly that of the comet over 3 nights. We conclude that the object is actually $1 \mathrm{P} / \mathrm{Halley}$.

In order to measure the flux of the object, each composite was scanned in $x$ and $y$, over $16^{\prime \prime}$ centered on the object, with a $0.2^{\prime \prime}$ step (=1 pixel). At each position, the flux was integrated over a circular aperture. The measurement presenting the highest flux is recorded, then the procedure is started over with a different aperture radius, up to $5^{\prime \prime}$, constituing a growth profile of the object. These growth profiles present a maximum $S / N$ in the $0.8-1.2^{\prime \prime}$ radius range. The corresponding flux is corrected for the aperture losses using the growth profile of well-exposed stars. The photometric profile of the total composite is displayed in Fig. 2. The profile is stellar within the error bars, indicating that no resolved coma is detected. It must be noted that, because of the large error bars, this is not a very constraining result.

The magnitudes are reported in Table 2. They are found in good agreement with the expected nuclear magnitude, given by

$V=13.95+5 \log (r \Delta)$

where 13.95 is the average nuclear magnitude obtained from the size and albedo determined by Keller et al. (1987), and considering that the color of the nucleus is the average value $V-R=0.44$ (Hainaut \& Delsanti 2002). As the solar phase angle was very small (cf. Table 3 ), its effect is small compared to the photometric errors. This indicate that the bare nucleus was 


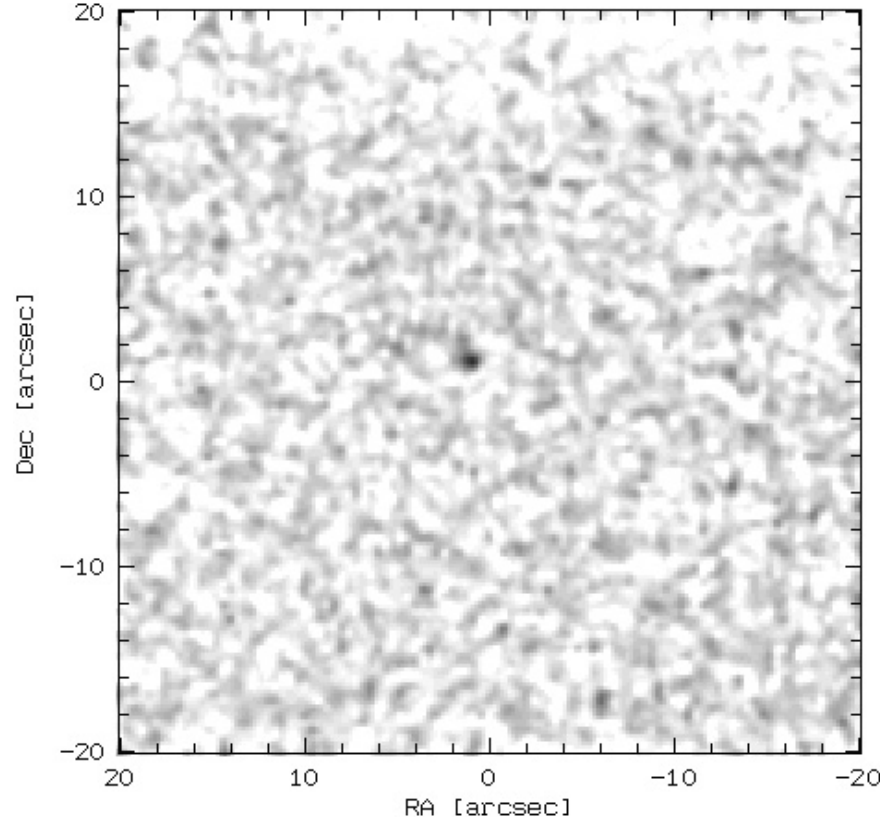

Fig. 1. Composite (totalling $32284 \mathrm{~s}, S / N=8$ ) of UT1, 3 and 4 exposures centered on the expected position of $1 \mathrm{P} / \mathrm{Halley}$. The object is visible $1.6^{\prime \prime} \mathrm{NW}$ of the predicted position.

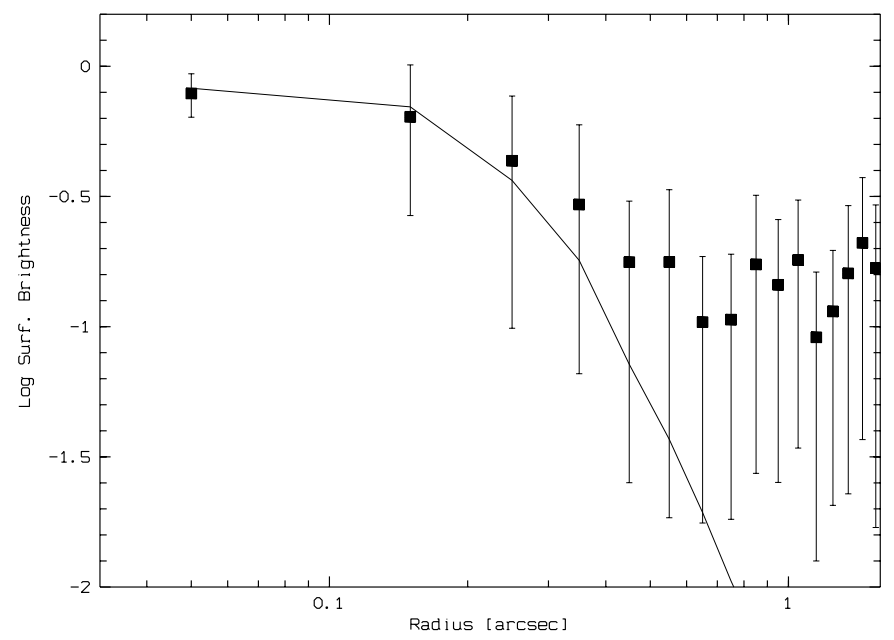

Fig. 2. Photometric profile of comet 1P/Halley (dots), measured on the $32284 \mathrm{~s}$ composite), compared to a mean stellar profile (line). The asymmetric error bars are a consequence of the logarithmic scale.

observed, with no cometary activity, as expected at the distance of the comet. Figure 3 displays the post-perihelion heliocentric lightcurve of the comet.

The position of the object is recorded on each composite as the $x, y$ having the best $S / N$, and is converted in $\alpha, \delta$ using the astrometric transformation used previously. The positions have been reported in Marsden (2003), and are listed in Table 3 together with the deviations from the ephemerides. The measurement uncertainty on the position of the comet is $\sim 1-2$ pix $=0.2-0.4^{\prime \prime}$, caused by the relatively low $S / N$ of the object. The residual of the astrometric calibration is $0.3^{\prime \prime} \mathrm{rms}$. Another source of error is the ephemerides, which are

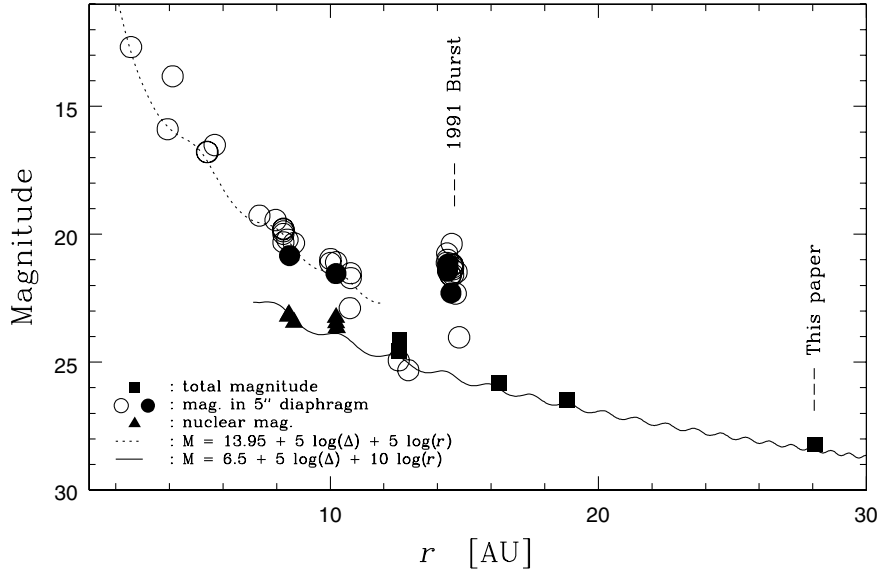

Fig. 3. Post-perihelion lightcurve of comet 1P/Halley. Open symbols are Kron-Cousin $R$ magnitudes from Meech (1999) (shifted by $V-R=$ 0.44); solid symbols are from Papers I-IV. Triangles are nuclear magnitudes; circle, magnitudes integrated in a $5^{\prime \prime}$ diameter aperture and squares, total magnitudes. The dashed line is a $r^{-4}$ curve adjusted to the circles (suggesting a possible behaviour of the active comet), and the solid line represents the mean nuclear magnitude (the variations being caused by the changes of geocentric distance).

mostly based on positions recorded with respect to other systems of reference star (e.g. SAO or PPM, depending on what was available at that time, compared to the current measurements calibrated with USNO-A). Because of all these effects, we conclude that the reported positions are compatible with the predictions.

\section{Summary}

Comet $1 \mathrm{P} /$ Halley has been observed at $r=28.1 \mathrm{AU}$, at the expected position and with the magnitude expected for the bare nucleus, as measured pre- and post-perihelion. The photometric profile of the object does not display any significant surface brightness excess compared to that of field stars. We conclude that no cometary activity was detected.

It is interesting to note that the same observations $(32000 \mathrm{~s}$ on VLT) would also have marginally detected the comet at aphelion (at $35 \mathrm{AU}$, with an expected magnitude $R=29.3$, we would still have a $\sim 3 \sigma$ detection), indicating that it is now feasible to follow this comet over its entire orbit.

Acknowledgements. We are grateful to R. Gilmozzi, director of VLT Paranal Observatory, for making some observing time available to this project with VIMOS on UT3. The very efficient support of S. Brillant on that instrument was a key to the success of these observations. We are also grateful to B. Marsden for computing updated orbital elements of the comet for the epoch of the observations, and to the Telescope and Instrument Operators for their skillful driving of the VLT.

\section{Appendix A: Observation log}

Table A.1 gives the detailed log of the observations. 
Table A.1. Log of the observations. Day and UT refer to the exposure epoch; Day is 2003 March. Exp. is the exposure time [s], T is the VLT Unit Telescope number, I the instrument (FORS1, FORS2, VIMOS), $F$ the filter used. AM is the airmass, M.Alt the altitude of the Moon (deg over the horizon) and M.Ph. the illuminated fraction, if the Moon is over the horizon. All the variables refer to the beginning of exposure. The seeing [arcsec, FWHM] is as measured on the frames.

\begin{tabular}{|c|c|c|c|c|c|c|c|}
\hline Day & UT & Exp. & $\mathrm{T} / \mathrm{I} / \mathrm{F}$ & $\mathrm{AM}$ & $\mathrm{S}$ & M.Alt & M.Ph \\
\hline 06 & $00: 50$ & 360 & $3 / \mathrm{VM} / \mathrm{R}$ & 1.22 & 1.5 & - & - \\
\hline 06 & $00: 59$ & 600 & $1 / \mathrm{F} 1 / \mathrm{V}$ & 1.19 & 1.8 & - & - \\
\hline 06 & $01: 22$ & 600 & $1 / \mathrm{F} 1 / \mathrm{V}$ & 1.15 & 1.7 & - & - \\
\hline 06 & $01: 56$ & 360 & $3 / \mathrm{VM} / \mathrm{R}$ & 1.11 & 1.1 & - & - \\
\hline 06 & 02:03 & 360 & $3 / \mathrm{VM} / \mathrm{R}$ & 1.11 & 1.1 & _- & - \\
\hline 06 & $02: 10$ & 360 & $3 / \mathrm{VM} / \mathrm{R}$ & 1.10 & 1.0 & - & - \\
\hline 06 & $02: 17$ & 360 & $3 / \mathrm{VM} / \mathrm{R}$ & 1.10 & 0.9 & - & - \\
\hline 06 & 02:24 & 360 & $3 / \mathrm{VM} / \mathrm{R}$ & 1.10 & 0.9 & - & - \\
\hline 06 & $02: 30$ & 360 & $3 / \mathrm{VM} / \mathrm{R}$ & 1.10 & 1.0 & - & - \\
\hline 06 & 02:37 & 360 & $3 / \mathrm{VM} / \mathrm{R}$ & 1.10 & 1.0 & - & - \\
\hline 06 & 02:44 & 360 & $3 / \mathrm{VM} / \mathrm{R}$ & 1.10 & 1.0 & - & - \\
\hline 06 & $02: 51$ & 360 & $3 / \mathrm{VM} / \mathrm{R}$ & 1.10 & 1.2 & - & - \\
\hline 06 & $02: 58$ & 360 & $3 / \mathrm{VM} / \mathrm{R}$ & 1.11 & 1.1 & - & - \\
\hline 07 & $00: 05$ & 600 & $1 / \mathrm{F} 1 / \mathrm{V}$ & 1.34 & 1.2 & 15.7 & 0.13 \\
\hline 07 & $00: 13$ & 360 & $3 / \mathrm{VM} / \mathrm{R}$ & 1.31 & 1.1 & 14.0 & 0.13 \\
\hline 07 & $00: 16$ & 600 & $1 / \mathrm{F} 1 / \mathrm{V}$ & 1.30 & 1.1 & 13.3 & 0.13 \\
\hline 07 & $00: 17$ & 360 & $4 / F 2 / R$ & 1.29 & 0.6 & 13.1 & 0.13 \\
\hline 07 & 00:20 & 358 & $3 / \mathrm{VM} / \mathrm{R}$ & 1.28 & 1.1 & 12.5 & 0.13 \\
\hline 07 & $00: 25$ & 360 & $4 / F 2 / R$ & 1.27 & 0.6 & 11.6 & 0.13 \\
\hline 07 & 00:27 & 360 & $3 / \mathrm{VM} / \mathrm{R}$ & 1.26 & 1.0 & 11.0 & 0.13 \\
\hline 07 & 00:27 & 600 & $1 / \mathrm{F} 1 / \mathrm{V}$ & 1.26 & 1.1 & 11.0 & 0.13 \\
\hline 07 & $00: 31$ & 360 & $4 / \mathrm{F} 2 / \mathrm{R}$ & 1.25 & 0.7 & 10.2 & 0.13 \\
\hline 07 & $00: 34$ & 360 & $3 / \mathrm{VM} / \mathrm{R}$ & 1.24 & 1.1 & 9.5 & 0.13 \\
\hline 07 & $00: 39$ & 360 & $4 / F 2 / R$ & 1.23 & 0.8 & 8.5 & 0.13 \\
\hline 07 & 00:40 & 600 & $1 / \mathrm{F} 1 / \mathrm{V}$ & 1.23 & 1.1 & 8.2 & 0.13 \\
\hline 07 & $00: 41$ & 360 & $3 / \mathrm{VM} / \mathrm{R}$ & 1.22 & 1.0 & 8.0 & 0.13 \\
\hline 07 & $00: 46$ & 360 & $4 / \mathrm{F} 2 / \mathrm{R}$ & 1.21 & 0.7 & 7.1 & 0.13 \\
\hline 07 & $00: 48$ & 360 & $3 / \mathrm{VM} / \mathrm{R}$ & 1.20 & 1.0 & 6.6 & 0.13 \\
\hline 07 & $00: 51$ & 600 & $1 / \mathrm{F} 1 / \mathrm{V}$ & 1.20 & 1.1 & 5.8 & 0.13 \\
\hline 07 & $00: 55$ & 360 & $3 / \mathrm{VM} / \mathrm{R}$ & 1.19 & 1.0 & 5.1 & 0.13 \\
\hline 07 & 01:02 & 362 & $3 / \mathrm{VM} / \mathrm{R}$ & 1.17 & 1.0 & 3.6 & 0.13 \\
\hline 07 & 01:02 & 600 & $1 / \mathrm{F} 1 / \mathrm{V}$ & 1.17 & 1.1 & 3.5 & 0.13 \\
\hline 07 & 01:07 & 360 & $4 / F 2 / R$ & 1.17 & 0.6 & 2.4 & 0.13 \\
\hline 07 & 01:09 & 360 & $3 / \mathrm{VM} / \mathrm{R}$ & 1.16 & 0.9 & 2.1 & 0.13 \\
\hline 07 & $01: 14$ & 360 & $4 / F 2 / R$ & 1.15 & 0.6 & 0.9 & 0.13 \\
\hline 07 & $01: 15$ & 360 & $3 / \mathrm{VM} / \mathrm{R}$ & 1.15 & 1.0 & 0.6 & 0.13 \\
\hline 07 & $01: 18$ & 600 & $1 / \mathrm{F} 1 / \mathrm{V}$ & 1.15 & 1.3 & - & - \\
\hline 07 & $01: 21$ & 360 & $4 / F 2 / R$ & 1.14 & 0.9 & - & - \\
\hline 07 & $01: 28$ & 360 & $4 / F 2 / R$ & 1.13 & 1.0 & - & - \\
\hline 07 & 01:29 & 360 & $3 / \mathrm{VM} / \mathrm{R}$ & 1.13 & 1.4 & - & - \\
\hline 07 & $01: 29$ & 600 & $1 / \mathrm{F} 1 / \mathrm{V}$ & 1.13 & 1.5 & - & - \\
\hline 07 & $01: 35$ & 360 & $4 / F 2 / R$ & 1.13 & 0.9 & - & - \\
\hline 07 & $01: 36$ & 361 & $3 / \mathrm{VM} / \mathrm{R}$ & 1.12 & 1.2 & - & - \\
\hline 07 & $01: 43$ & 360 & $3 / \mathrm{VM} / \mathrm{R}$ & 1.12 & 1.1 & - & - \\
\hline 07 & 01:57 & 360 & $3 / \mathrm{VM} / \mathrm{R}$ & 1.11 & 1.0 & - & - \\
\hline 07 & 02:03 & 360 & $3 / \mathrm{VM} / \mathrm{R}$ & 1.10 & 1.0 & - & - \\
\hline 07 & 02:10 & 362 & $3 / \mathrm{VM} / \mathrm{R}$ & 1.10 & 1.1 & - & - \\
\hline 07 & $02: 17$ & 360 & $3 / \mathrm{VM} / \mathrm{R}$ & 1.10 & 1.0 & - & - \\
\hline 08 & $00: 13$ & 360 & $3 / \mathrm{VM} / \mathrm{R}$ & 1.30 & 1.0 & 20.2 & 0.17 \\
\hline 08 & $00: 18$ & 360 & $4 / F 2 / R$ & 1.28 & 0.6 & 19.1 & 0.17 \\
\hline 08 & 00:20 & 360 & $3 / \mathrm{VM} / \mathrm{R}$ & 1.27 & 1.0 & 18.8 & 0.17 \\
\hline 08 & $00: 25$ & 360 & $4 / \mathrm{F} 2 / \mathrm{R}$ & 1.25 & 0.5 & 17.6 & 0.17 \\
\hline 08 & $00: 26$ & 360 & $3 / \mathrm{VM} / \mathrm{R}$ & 1.25 & 0.9 & 17.4 & 0.17 \\
\hline 08 & $00: 32$ & 360 & $4 / F 2 / R$ & 1.24 & 0.5 & 16.2 & 0.17 \\
\hline 08 & 00:33 & 360 & $3 / \mathrm{VM} / \mathrm{R}$ & 1.23 & 1.0 & 16.0 & 0.17 \\
\hline 08 & 00:40 & 360 & $3 / \mathrm{VM} / \mathrm{R}$ & 1.21 & 1.0 & 14.6 & 0.17 \\
\hline 08 & $00: 41$ & 360 & $4 / F 2 / R$ & 1.21 & 0.6 & 14.5 & 0.17 \\
\hline 08 & $00: 47$ & 360 & $3 / \mathrm{VM} / \mathrm{R}$ & 1.20 & 1.1 & 13.2 & 0.17 \\
\hline 08 & $00: 47$ & 360 & $4 / F 2 / R$ & 1.20 & 0.7 & 13.2 & 0.17 \\
\hline 08 & $00: 54$ & 360 & $3 / \mathrm{VM} / \mathrm{R}$ & 1.18 & 1.3 & 11.8 & 0.17 \\
\hline 08 & 00:54 & 360 & $4 / \mathrm{F} 2 / \mathrm{R}$ & 1.18 & 0.9 & 11.7 & 0.17 \\
\hline 08 & 01:01 & 360 & $3 / \mathrm{VM} / \mathrm{R}$ & 1.17 & 1.2 & 10.4 & 0.17 \\
\hline 08 & 01:02 & 360 & $4 / F 2 / R$ & 1.17 & 0.7 & 10.1 & 0.17 \\
\hline 08 & 01:02 & 600 & $1 / \mathrm{F} 1 / \mathrm{V}$ & 1.17 & 1.2 & 10.1 & 0.17 \\
\hline 08 & 01:07 & 360 & $3 / \mathrm{VM} / \mathrm{R}$ & 1.16 & 1.1 & 9.0 & 0.17 \\
\hline 08 & 01:09 & 360 & $4 / F 2 / R$ & 1.16 & 0.7 & 8.6 & 0.17 \\
\hline 08 & $01: 14$ & 360 & $3 / \mathrm{VM} / \mathrm{R}$ & 1.15 & 1.0 & 7.5 & 0.17 \\
\hline 08 & $01: 16$ & 360 & $4 / F 2 / R$ & 1.14 & 0.7 & 7.2 & 0.17 \\
\hline 08 & $01: 27$ & 600 & $1 / \mathrm{F} 1 / \mathrm{V}$ & 1.13 & 1.2 & 4.9 & 0.17 \\
\hline 08 & $01: 32$ & 360 & 4/F2/R & 1.13 & 0.7 & 3.8 & 0.17 \\
\hline 08 & $01: 38$ & 360 & $3 / \mathrm{VM} / \mathrm{R}$ & 1.12 & 1.1 & 2.5 & 0.17 \\
\hline 08 & $01: 38$ & 600 & $1 / \mathrm{F} 1 / \mathrm{V}$ & 1.12 & 1.1 & 2.6 & 0.17 \\
\hline 08 & 01:39 & 360 & $4 / F 2 / R$ & 1.12 & 0.6 & 2.3 & 0.17 \\
\hline 08 & $01: 45$ & 360 & $3 / \mathrm{VM} / \mathrm{R}$ & 1.11 & 1.0 & 1.0 & 0.17 \\
\hline 08 & $01: 52$ & 360 & $3 / \mathrm{VM} / \mathrm{R}$ & 1.11 & 1.0 & - & - \\
\hline 08 & 01:59 & 360 & $3 / \mathrm{VM} / \mathrm{R}$ & 1.10 & 1.0 & - & - \\
\hline 08 & 02:06 & 360 & $3 / \mathrm{VM} / \mathrm{R}$ & 1.10 & 1.0 & - & - \\
\hline 08 & $02: 13$ & 360 & $3 / \mathrm{VM} / \mathrm{R}$ & 1.10 & 1.1 & - & - \\
\hline 08 & $02: 20$ & 360 & $3 / \mathrm{VM} / \mathrm{R}$ & 1.10 & 1.1 & - & - \\
\hline 08 & $02: 26$ & 360 & $3 / \mathrm{VM} / \mathrm{R}$ & 1.10 & 1.4 & - & - \\
\hline 08 & $02: 33$ & 360 & $3 / \mathrm{VM} / \mathrm{R}$ & 1.10 & 1.2 & - & - \\
\hline 08 & $02: 40$ & 360 & $3 / \mathrm{VM} / \mathrm{R}$ & 1.10 & 1.1 & - & - \\
\hline
\end{tabular}




\section{References}

Banse, K., Ponz, D., Ounnas, C., Grosbol, P., \& Warmels, R. 1988, in The Ninth Santa Cruz Summer Workshop in Astronomy and Astrophysics, ed. L. B. Robinson (New York: Springer-Verlag), 431 Bertin, E., \& Arnout, S. 1996, A\&AS, 117, 393

ESO 1999, MIDAS: Munich Image Data Analysis System, http://www. eso.org/projects/esomidas

Hainaut, O. R., Delahodde, C. E., Böhnhardt, H., et al. 2000, A\&A, 356, 1076

Hainaut, O. R., \& Delsanti, A. 2002, A\&A, 389, 641

Hainaut, O. R., Meech, K. J., Böhnhardt, H., \& West, R. M. 1998, A\&A, 333, 746

Hainaut, O. R., West, R. M., Marsden, B. G., Smette, A., \& Meech, K. J. 1995, A\&A, 293, 941

Keller, H. U., Delamere, W. A., Reitsema, H. J., Huebner, W. F., \& Schmidt, H. U. 1987, A\&A, 187, 807

Marsden, B. G. 2003, MPE Circ., 48
Meech, K., \& Hainaut, O. 2001, Tidal and Collisional Interactions of Small Bodies in the Solar System, ed. M. Ya. Marov, \& H. Rickman (Dordrecht: Astrophysics and Space Science Library, Kluwer), distant Comets Observations, 1

Meech, K. J. 1991, Comets in the Post-Halley Era, ed. R. L. Newburn, M. Neugebauer, \& J. Rahe (Dordrecht: Kluwer), 629

Meech, K. J. 1999, Evolution and Source Regions of Asteroids and Comets, ed. J. Svoren, E. M. Pittich, \& H. Rickman, 195

Meech, K. J. 2003, Asteroids, Comets, Meteors 1996, ed. A. C. Levasseur-Regourd, in press since 1997, preprint available from the author

Meech, K. J., Hainaut, O. R., \& Marsden, B. G. 2000, in Minor Bodies in the Outer Solar System, ed. Fitzsimmons, Jewitt, \& West (Springer), 75

Meech, K. J., Hainaut, O. R., \& Marsden, B. G. 2003, Icarus, in press Walter, X., \& West, R. M. 1986, A\&A, 228, 1

West, R. M. 1990, A\&A, 228, 532

West, R. M., Hainaut, O. R., \& Smette, A. 1991, A\&A, 246, L77

West, R. M., \& Jørgensen, H. E. 1989, A\&A, 217, 307 